\title{
Observation of Glucose Metabolism in the Thalamic Nuclei by Fusion PET/MRI
}

\author{
Zang-Hee Cho ${ }^{1,2}$, Young-Don Son ${ }^{1}$, Hang-Keun Kim ${ }^{1}$, Nam-Beom Kim ${ }^{1}$, Eun-Jung Choi ${ }^{1}$, Sang-Yoon Lee ${ }^{1}$, \\ Je-Geun Chi ${ }^{3}$, Chan-Woong Park ${ }^{1}$, Young-Bo Kim ${ }^{1}$, and Seiji Ogawa ${ }^{1,4}$ \\ ${ }^{1}$ Neuroscience Research Institute, Gachon University of Medicine and Science, Incheon, South Korea; ${ }^{2}$ Department of Radiological \\ Sciences, University of California, Irvine, California; ${ }^{3}$ Department of Pathology, Seoul National University, Seoul, South Korea; and \\ ${ }^{4}$ Ogawa Laboratories for Brain Function Research, Hamano Life Science Research Foundation, Tokyo, Japan
}

\begin{abstract}
The anatomy of the thalamus and its connectivity with surrounding areas are known. Localized metabolic activities at the thalamic substructural level have not been measured in vivo in human brains because of limited resolution and contrast. Methods: The energy metabolism and fine anatomic structures of the thalamus were measured simultaneously in 5 healthy subjects using a PET/MRI fusion imaging system. Measured metabolism in individual thalamic nuclei was quantified by corresponding PET/MRI images. Results: Substructures of the thalamus were clearly distinguished in 7.0-T MRI images, and the corresponding metabolic activities measured by PET were integrated by the PET/MRI system. The medial dorsal thalamic nucleus consistently showed the highest glucose uptake among the thalamic nuclei. Conclusion: These results demonstrate that substructure-specific metabolic activities in the thalamus can be measured with a PET/MRI system consisting of an ultrahigh-resolution PET component and an ultra-high-field MRI component.
\end{abstract}

Key Words: metabolic activities in the thalamus; PET/MRI; fusion imaging

J Nucl Med 2011; 52:401-404

DOI: 10.2967/jnumed.110.081281

\section{$\mathbf{R}$}

ecent advances in ultra-high-field MRI allow visualization of the substructures of the hippocampus in the human brain $(1,2)$. Concurrently, in vivo human molecular imaging devices, such as PET, have also been markedly improved (3). With these advances, PET/MRI fusion systems have been developed for combined imaging of structural and molecular information on humans in vivo (4-6). The first development in this approach was separate PET and MRI scanners coupled via a shuttle bed (4), which mechanically identified the imaging coordinates of both scanners with submillimeter accuracy $(<100 \mu \mathrm{m})$. Another development was an insertion-type scanner in which the

\footnotetext{
Received Jul. 13, 2010; revision accepted Nov. 18, 2010.

For correspondence or reprints contact: Zang-Hee Cho, Neuroscience Research Institute, Gachon University of Medicine and Science, 1198 KuwolDong, Namdong-Gu, Incheon, 405-760, South Korea.

E-mail: zcho@gachon.ac.kr

COPYRIGHT @ 2011 by the Society of Nuclear Medicine, Inc.
}

PET detectors were inserted into the MRI scanner $(5,6)$. The latter has the potential to provide true simultaneous scanning, whereas the former provides spatially accurate, high-resolution images in a near real-time simultaneous scan by incorporating the 2 highest-end scanners currently available: the High-Resolution Research Tomograph, or HRRT (CTI-Siemens), which is dedicated to human brain research, and the ultra-high-field 7T MRI scanner (Siemens).

The thalamus is a suitable and attractive candidate for this PET/MRI system because a system capable of high spatial resolution is required to resolve the many substructures, each of which has substantially diverse functions, within the small volume of the thalamus. The present study demonstrated that the new human brain PET/MRI system (4) can be used in vivo in humans to anatomically distinguish the individual thalamic nuclei and to measure their corresponding glucose metabolism.

\section{MATERIALS AND METHODS}

\section{PET/MRI System}

All images were obtained using the HRRT/7T MRI system that we recently developed (4). This system consists of 3 major components: the HRRT, the 7T MRI scanner, and the shuttle bed. The HRRT is a new-generation ultra-high-resolution PET system that can image at an isovoxel resolution of as high as 2.5 $\mathrm{mm}$ in full width half maximum. It has a transaxial diameter of $46.9 \mathrm{~cm}$ and an axial field of view of $25.2 \mathrm{~cm}$, enough to cover the whole brain. The small system diameter (transaxial) of the HRRT gives a substantially increased solid angle - thus its high sensitivity. In addition, the HRRT uses the smallest detector currently available $(2.3 \times 2.3 \mathrm{~mm})$ to obtain the highest resolution possible (3). On the other front, the 7T MRI scanner has an in-plane resolution capability of $250 \mu \mathrm{m}$-hitherto unavailable from any other human whole-body MRI system (4). The combination of the 2 modalities has enabled us to image metabolic functions of many suborgans (e.g., hippocampus) in the human brain in vivo (1).

A shuttle bed added to the HRRT and 7T MRI scanner allows us to integrate the 2 modalities into a single mode, PET/MRI, similar to PET/CT. After MRI, the shuttle bed rotates $180^{\circ}$ clockwise or counterclockwise at the middle of the system to allow a brain scan at PET, because both the HRRT and the 7T MRI scanner are 
brain-dedicated and the whole body is unable to pass through the scanners (the bore size of the HRRT is about $30 \mathrm{~cm}$ ). All components of the shuttle bed are nonmagnetic and can withstand the high-fringe field of the 7.0-T magnet.

\section{Subjects}

Five healthy volunteers were recruited and provided written informed consent. The experiments and informed consent form were approved by the Institutional Review Board of the Gachon University of Medicine and Science and by the Korea Food and Drug Administration.

\section{Scanning Protocol}

A bolus injection of ${ }^{18} \mathrm{~F}-\mathrm{FDG}$ (185 MBq) was given before MRI began. The subjects were asked to close their eyes during MRI, which was then performed for 30 min using a T1-weighted 3-dimensional (3D) magnetization-prepared rapid-acquisition gradient-echo sequence, with a recovery time of 4,000 ms, echo time of $5.34 \mathrm{~ms}$, inversion time of $1,000 \mathrm{~ms}$, and flip angle of $10^{\circ}$, to obtain 160 coronal $0.33 \times 0.33 \times 1.2 \mathrm{~mm}$ slices. Afterward, the shuttle bed was transported to the PET scanner, and PET was conducted for $30 \mathrm{~min}$. Additional transmission scans using the ${ }^{137} \mathrm{Cs}$ point source at the HRRT were conducted for attenuation correction. The PET images were reconstructed using 3D ordinary Poisson ordered-subset expectation maximization accelerated by symmetry and single-instruction multipledata-based projection and backprojection algorithms (7). The reconstructed image had a $256 \times 256 \times 207$ matrix with a nominal isovoxel of $1.22 \times 1.22 \times 1.22 \mathrm{~mm}$. After scanning was completed, the scanned MRI and PET images were then automatically integrated to the same coordinates by the shuttle bed. No additional image processing or coregistration was needed for image fusion. This precise image fusion based on the submillimeter-precision mechanical shuttle bed is a key ingredient of the PET/MRI system.

\section{Data Analysis}

The standardized uptake value ratio was obtained by normalizing the uptake value of PET images by the uptake value of each subject's cerebellum. Based on a published anatomic reference (8), 16 regions of interest (ROIs) - that is, anterior thalamic nucleus, medial dorsal thalamic nucleus, centromedian thalamic nucleus, dorsal superficial nucleus, ventral anterior thalamic nucleus, ventral lateral thalamic nucleus, ventral lateral posterior thalamic nucleus, and pulvinar thalami, bilaterally—were selected and manually drawn on the acquired MRI images using a 3D volume-of-interest tool (Vinci; Max Planck Institute). Finally, the standardized uptake value ratio of each ROI was obtained from the corresponding PET image for quantitative analysis. This paper also uses the terminology of the anatomic reference (8).

\section{RESULTS}

For MRI, the T1-weighted 3D magnetization-prepared rapid-acquisition gradient-echo sequence was chosen in order to obtain fine, submillimeter, structural images of the thalamus. An example of in vivo images of the thalamus obtained with the 7T MRI scanner is shown in Figure 1A. Many substructures of the thalamus are clearly distinguishable, such as the pulvinar thalami, ventral lateral thalamic nucleus, ventral posterior lateral thalamic nucleus, centro- median thalamic nucleus, and medial dorsal thalamic nucleus, as well as surrounding landmark nuclei such as the putamen and caudate nucleus (Fig. 1D). Corresponding images of metabolic activity were also obtained by ${ }^{18} \mathrm{~F}-$ FDG HRRT (Fig. 1B). When the images were fused, functional correspondence was clearly identified for the caudate nucleus, putamen, dorsal superficial nucleus, pulvinar thalami, ventral lateral thalamic nucleus, centromedian thalamic nucleus, medial dorsal thalamic nucleus, and red nucleus. Figure $1 \mathrm{C}$ is the corresponding PET/MRI image. To demonstrate the capability of image fusion for the whole thalamic area, we selected 5 representative slices; the corresponding glucose metabolism is shown in Figure 2 (Supplemental Fig. 1 shows the whole thalamus; supplemental materials are available online only at http://jnm.snmjournals. org). To show the relative metabolic activities in the various nuclei, we manually drew 16 ROIs along the longest axis of the thalamus (anterior to posterior) and measured their activities as shown in Figure 3. Although the boundaries of some nuclei are not clear enough for the parcellation, the high image contrast obtained with the 7T MRI scanner clearly showed the boundaries of the major nuclei. We found that the medial dorsal thalamic nucleus had the highest glucose uptake. When we evaluated the 5 participating subjects, all 5 showed the same results (Supplemental Fig. 2).

\section{DISCUSSION}

The high-magnetic-field 7T MRI scanner enabled us to show the various thalamic nuclei clearly. Above all, the high magnetic field produces images of high signalto-noise ratio with high contrast between tissues. Clear delineation of the thalamic nuclei enabled us to parcel each ROI and measure the glucose metabolism within the parceled regions. In addition to the ultra-high resolution and contrast of the 7T MRI images, the high-resolution capability of the HRRT was a key factor in improving the accuracy of metabolism measurement for each subnucleus by reducing the partial-volume effect, although still not meeting the MRI resolution. If the HRRT image were to have a higher resolution, the partial-volume effect could be reduced further (8) and the quantitative accuracy of the data could thus be improved, especially for a small ROI. This synergistic operation of the HRRT/7T MRI scanner is probably the one capability that distinguishes it from the other PET/MRI systems that have low resolution (PET) and a low magnetic field (MRI).

Consistently higher glucose uptake was shown for the medial dorsal thalamic nucleus, dorsal superficial nucleus, and pulvinar thalami than for the other thalamic nuclei. This result is consistent with previous findings that the cell density of the medial and the posterior thalamus is higher than that of the anterior and the lateral thalamus (9). One can assume that the higher density of the cell body, therefore, is related to the higher glucose uptake in the PET image. 


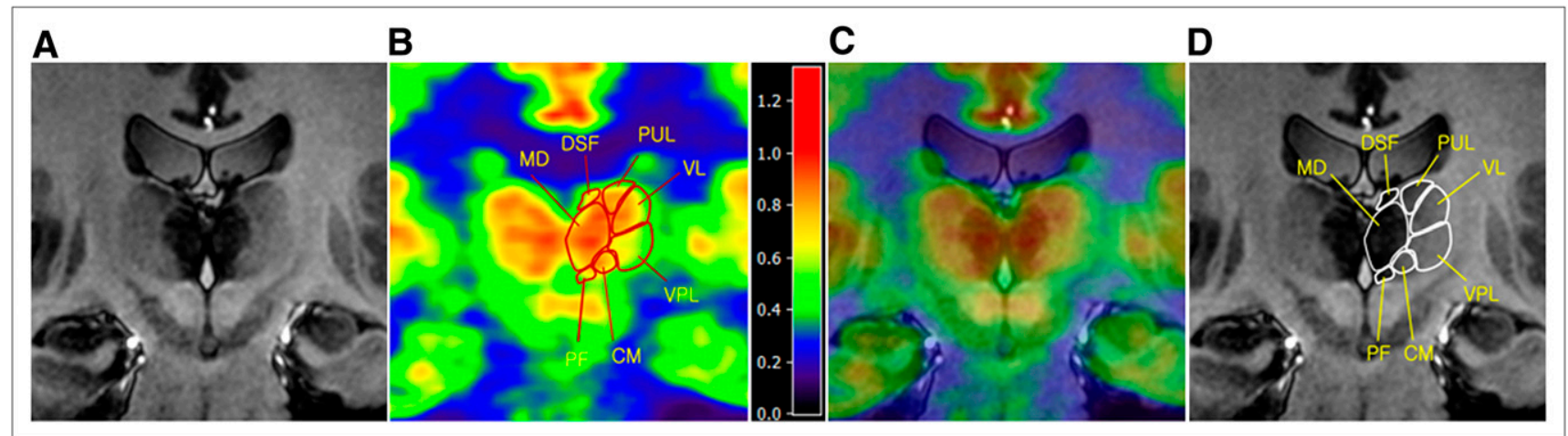

FIGURE 1. PET/MRI image of selected coronal section of thalamus: $7 T$ MRI image of thalamus with various thalamic nuclei (A), HRRT image of ${ }^{18} \mathrm{~F}-\mathrm{FDG}$ glucose metabolism (B), PET/MRI image with labeling (C), and selected ROls and labels based on $7 \mathrm{~T}$ MRI image (D). These correspond to slice 3 in Figure 2. CM = centromedian thalamic nucleus; DSF = dorsal superficial nucleus; MD = medial dorsal thalamic nucleus; $\mathrm{PF}=$ parafascicular thalamic nucleus; $\mathrm{PUL}=$ pulvinar thalami; $\mathrm{VL}=$ ventral lateral thalamic nucleus; $\mathrm{VPL}=\mathrm{ventral}$ lateral posterior thalamic nucleus.

Functional metabolic images obtained by the PET/MRI system could be an important asset for neuroscience research because the thalamus has such an important role in brain function and in the mechanisms of pain and of diseases such as schizophrenia and epilepsy. We envision that this study can be extended to other uses of PET for molecular imaging, such as for imaging $\mathrm{D}_{2}$ receptor distri- bution within each thalamic nucleus. This study also supports the future potential of using PET/MRI in neuroscience research and clinical neurology.

\section{CONCLUSION}

The high-resolution, high-contrast images of the 7T MRI scanner enabled us to distinguish the thalamic nuclei in

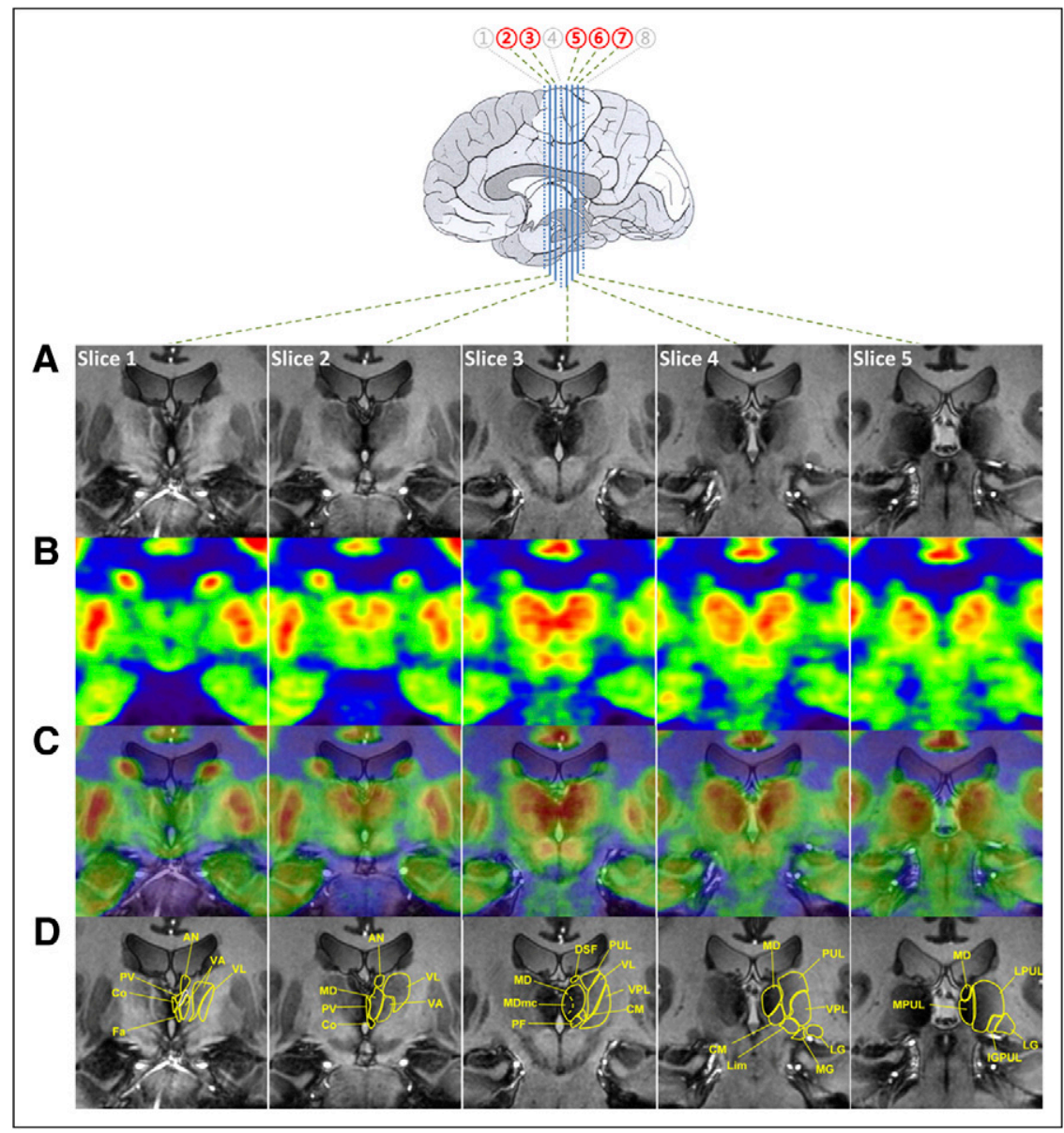

FIGURE 2. Five selected PET/MRI slices of thalamus of single subject: coronal $7 \mathrm{~T}$ MRI images of thalamic nuclei (A), HRRT images of ${ }^{18} \mathrm{~F}-\mathrm{FDG}$ glucose metabolism (B), PET/MRI images (C), and selected ROls and labels based on 7T MRI (D). Total slices are shown in Supplemental Figure 1; upper number is slice number of Supplemental Figure 1. $\mathrm{AN}=$ anterior thalamic nucleus; $\mathrm{CM}=$ centromedian thalamic nucleus; $\mathrm{Co}=$ commissural nucleus; DSF = dorsal superficial nucleus; $\mathrm{Fa}=$ fasciculosus nucleus; IGPUL = intergeniculate pulvinar thalami; LG = lateral geniculate nucleus; $\mathrm{Lim}=$ limitans nucleus; LPUL = lateral pulvinar thalami nucleus; $M D=$ medial dorsal thalamic nucleus; $\mathrm{MDmc}=$ medial dorsal thalamic nucleus, magnocellular part; $M G=$ medial geniculate nucleus; MPUL $=$ medial pulvinar thalami nucleus; $P F=$ parafascicular thalamic nucleus; PUL = pulvinar thalami; $\mathrm{PV}=$ paraventricular thalamic nucleus; $\mathrm{VA}=$ ventral anterior thalamic nucleus; $\mathrm{VL}=$ ventral lateral thalamic nucleus; $\mathrm{VPL}=$ ventral lateral posterior thalamic nucleus. (Full data are in Supplemental Fig. 1.) 


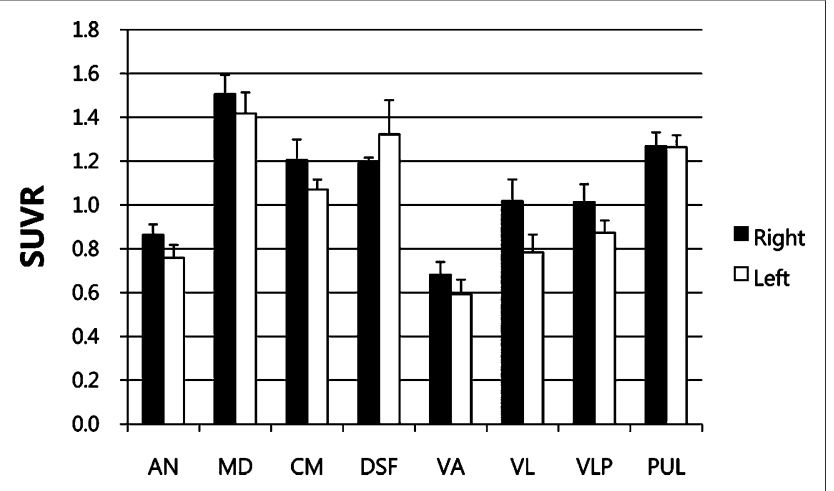

FIGURE 3. Quantified (averaged) glucose metabolism of various thalamic nuclei obtained from 5 subjects.

detail, thereby allowing localization of the corresponding in vivo metabolic activities obtained by the HRRT. We have demonstrated, for the first time, that quantitative subnucleus glucose metabolism is well aligned with the corresponding anatomic designation of the thalamus. This correspondence was shown, in vivo, by a PET/MRI system consisting of 2 ultra-high-resolution imaging devices, the brain-dedicated HRRT and the 7T MRI scanner.

\section{ACKNOWLEDGMENTS}

This work was supported by grant 2008-04159 from the National Research Foundation (NRF) and grant R332009000101190 from the Korea Science and Engineering Foundation (KOSEF), funded by the Ministry of Education, Science and Technology, South Korea.

\section{REFERENCES}

1. Cho ZH, Han JY, Hwang SI, et al. Quantitative analysis of the hippocampus using images obtained from 7.0 T MRI. Neuroimage. 2010;49:2134-2140.

2. Blow N. Neuroscience tools: brain insights. Nat Methods. 2008;5:981-987.

3. Wienhard K, Schmand M, Casey ME, et al. The ECAT HRRT: performance and first clinical application of the new high resolution research tomograph. IEEE Trans Nucl Sci. 2002;49:104-110.

4. Cho ZH, Son YD, Kim HK, et al. A fusion PET-MRI system with a high-resolution research tomograph-PET and ultra-high field 7.0T-MRI for the moleculargenetic imaging of the brain. Proteomics. 2008;8:1302-1323.

5. Judenhofer MS, Wehrl HF, Newport DF, et al. Simultaneous PET-MRI: a new approach for functional and morphological imaging. Nat Med. 2008;14:459-465.

6. Evanko D. Two pictures are better than one. Nat Methods. 2008;5:377.

7. Hong IK, Chung ST, Kim HK, Kim YB, Son YD, Cho ZH. Ultra fast symmetry and SIMD-based projection-backprojection (SSP) algorithm for 3-D PET image reconstruction. IEEE Trans Med Imaging. 2007;26:789-803.

8. van Velden FH, Kloet RW, van Berckel BN, et al. HRRT versus HR + human brain PET studies: an interscanner test-retest study. J Nucl Med. 2009;50:693-702.

9. Mai JK, Assheuer J, Paxinos G. Atlas of the Human Brain. San Diego, CA: Academic Press; 1997. 